BGU-PH-98/02

CERN-TH/98-53

hep-th/9803018

\title{
Duality in Cosmological Perturbation Theory
}

\author{
R. Brustein ${ }^{1,2}$, M. Gasperini ${ }^{3,2}$ and G. Veneziano ${ }^{2}$ \\ (1) Department of Physics, Ben-Gurion University, Beer-Sheva 84105, Israel \\ (2) Theory Division, CERN, CH-1211 Geneva 23, Switzerland \\ (3) Dipartimento di Fisica Teorica, Università di Torino, Via P. Giuria 1, 10125 Turin, Italy \\ and Istituto Nazionale di Fisica Nucleare, Sezione di Torino, Turin, Italy \\ E-mail: ramyb@bgumail.bgu.ac.il, gasperini@to.infn.it, venezia@nxth04.cern.ch
}

\begin{abstract}
Cosmological perturbation equations derived from low-energy effective actions are shown to be invariant under a duality transformation reminiscent of electric-magnetic, strong-weak coupling, S-duality. A manifestly dualityinvariant approximation for perturbations far outside the horizon is introduced, and it is argued to be useful even during a high curvature epoch. Duality manifests itself through a remnant symmetry acting on the classical moduli of cosmological models, and implying lower bounds on the number and energy density of produced particles.
\end{abstract}

Typeset using REVTEX 


\section{INTRODUCTION}

In inflationary cosmology [1] the Universe quickly becomes homogeneous, isotropic, and spatially flat, but quantum mechanics superimposes on the smooth classical backgrounds calculable inhomogeneity perturbations [2]. It is the purpose of this paper to point out a general duality symmetry obeyed by the lowest-order equations for these perturbations, and to show that duality may be used to obtain lower bounds on the energy density of the produced particles.

Our analysis applies to any physical perturbation $\Psi$ that is described, to lowest order in the strength of the perturbation and in its derivatives, by the following quadratic action

$$
I=\frac{1}{2} \int d \eta d^{3} x S(\eta)\left[\Psi^{\prime 2}-(\nabla \Psi)^{2}\right]
$$

In eq. (1.1), which is generic in low-energy spatially flat backgrounds, $\eta$ is the conformal time coordinate, and a prime denotes $\partial / \partial \eta$. The function $S(\eta)$, sometimes called the "pump" field, is, for any given $\Psi$, a known function of the scale factor, $a(\eta)$, and of additional scalar fields (such as a dilaton $\phi(\eta)$, moduli $b_{i}(\eta)$, etc.) on which the background may depend.

The duality symmetry, which, to the best of our knowledge, has not been noticed before in the context of cosmological perturbation theory, involves the interchange of the perturbation $\Psi$ with its conjugate momentum $\Pi$, as well as the inversion of the pump field $S$. In some cases it corresponds to a time-dependent version of $T$-duality, interchanging small and large scale factors [3,4]; in others it corresponds to a time-dependent version of $S$-duality [5,6], interchanging weak and strong coupling and electric and magnetic degrees of freedom; in most cases it amounts to a time-dependent mixture of both. As in other applications, duality turns out to be a powerful tool for obtaining results that are hard to obtain otherwise.

Although most of our discussion will be completely general, it may be useful to recall that, in the context of string cosmology, the massless perturbation $\Psi$ may represent a physical polarization of tensor metric perturbations (i.e. a gravity wave), physical electromagnetic perturbations (i.e. photons), axionic perturbations, and so on. In these three cases the pump 
field $S$ corresponds, respectively, to the Einstein-frame scale factor [0,8], given in terms of the dilaton as $S=a^{2} e^{-\phi}$, to the inverse string coupling [9] $S=e^{-\phi}$, and to the "axion-frame" scale factor [10] $S=a^{2} e^{\phi}$. Here, and in what follows, $a$ is the scale factor of the so-called string-frame metric, which coincides with the usual scale factor of general relativity whenever the dilaton is constant. For a generic string cosmology perturbation $S(\eta)=a^{2 m} e^{l \phi} b_{i}^{2 m_{i}}$, where the numbers $l, m, m_{i}$ depend on the type of perturbation. Arguably, the action (1.1) provides a useful description for a much wider class of perturbations.

\section{DUALITY}

In order to discuss the duality symmetry of the action (1.1), it is convenient to use the Hamiltonian formalism. The Hamiltonian corresponding to (1.1) is given by

$$
H=\frac{1}{2} \int d^{3} x\left[S^{-1} \Pi^{2}+S(\nabla \Psi)^{2}\right],
$$

where $\Pi$ is the momentum conjugate to $\Psi$. The first-order Hamilton equations read

$$
\Psi^{\prime}=\frac{\delta H}{\delta \Pi}=S^{-1} \Pi, \quad \Pi^{\prime}=-\frac{\delta H}{\delta \Psi}=S \nabla^{2} \Psi
$$

and lead to the decoupled second-order equations

$$
\Psi^{\prime \prime}+\frac{S^{\prime}}{S} \Psi^{\prime}-\nabla^{2} \Psi=0, \quad \Pi^{\prime \prime}-\frac{S^{\prime}}{S} \Pi^{\prime}-\nabla^{2} \Pi=0
$$

of which only the first is commonly used in the study of perturbations. In Fourier space the Hamiltonian (2.1) is given by

$$
H=\frac{1}{2} \sum_{\vec{k}}\left(S^{-1} \Pi_{\vec{k}} \Pi_{-\vec{k}}+S k^{2} \Psi_{\vec{k}} \Psi_{-\vec{k}}\right),
$$

where $\Psi_{-\vec{k}}=\Psi_{\vec{k}}^{*}$ and $\Pi_{-\vec{k}}=\Pi_{\vec{k}}^{*}$. The equations of motion become

$$
\Psi_{\vec{k}}^{\prime}=S^{-1} \Pi_{-\vec{k}}, \quad \quad \Pi_{\vec{k}}^{\prime}=-S k^{2} \Psi_{-\vec{k}},
$$

where $k=|\vec{k}|$. The transformation 


$$
\Pi_{\vec{k}} \rightarrow \widetilde{\Pi}_{\vec{k}}=k \Psi_{\vec{k}}, \quad \Psi_{\vec{k}} \rightarrow \widetilde{\Psi}_{\vec{k}}=-k^{-1} \Pi_{\vec{k}}, \quad S \rightarrow \widetilde{S}=S^{-1}
$$

leaves the Hamiltonian, Poisson brackets, and equations of motion unchanged.

We shall call this transformation, for short, $S$-duality since it contains the usual strongweak coupling duality as a special case. Indeed, by perturbing the tree-level, lowestderivative string effective action, one finds that, for heterotic, four-dimensional gauge bosons, the function $S$ is given simply by [9] $S(\eta)=e^{-\phi(\eta)}$. Since $e^{\phi}$ determines the effective string coupling constant, $e^{\phi}=g_{\text {string }}^{2}$, the transformation $S \rightarrow \widetilde{S}=S^{-1}$ amounts to performing, at each time $\eta$, a strong-weak coupling transformation $g_{\text {string }} \rightarrow g_{\text {string }}^{-1}$, which is part of the $S L(2, Z) S$-duality group [5]. Moreover, in the case of electromagnetic perturbations, the contribution of $k \Psi_{k}$ to the Hamiltonian can be identified with that of the magnetic field, whereas $\Pi$ is proportional to the electric field, hence, in this case, the transformation (2.6) exactly coincides with electric-magnetic duality.

We are interested in a situation where the time evolution of the pump field amplifies the zero-point vacuum fluctuations of the field $\Psi$, satisfying the initial conditions

$$
\left\langle S^{-1} \Pi^{2}\right\rangle=\left\langle S(\nabla \Psi)^{2}\right\rangle
$$

where $\langle\cdots\rangle$ denotes ensemble average, or expectation value if perturbations are quantized. Under $S$-duality the equations of motion are form-invariant, while the initial conditions are invariant on the average in the sense of eq. (2.7). Therefore, under $S$-duality, solutions of the perturbation equations properly normalized to zero-point fluctuations, are mapped into other normalized solutions with the same total Hamiltonian. We will show that, as a consequence of this property, the energy spectrum can be consistently estimated by truncating the solutions outside the horizon to the frozen modes of $\Psi$ and $\Pi$.

This may be regarded as trivial, if one has in mind a background where the non-frozen part of $\Psi$ quickly decays outside the horizon, as in the standard inflationary scenario [2]. There are backgrounds, however, in which $\Psi$ grows in time instead of decaying, and the growing mode of $\Psi$ dominates the perturbation spectrum. Typical examples can be found in scalar-tensor models of gravity and, in particular, in string cosmology [11]. In this context, 
the possibility of a consistent truncation to the frozen part of the perturbations is a powerful result. Only in a duality-invariant approach is such a truncation consistent, since, as shown below, the contribution to the Hamiltonian of the growing mode of $\Psi$ is simply replaced by the contribution of the frozen part of its duality-related conjugate momentum.

\section{APPROXIMATE SOLUTIONS INSIDE AND OUTSIDE THE HORIZON}

In order to solve the perturbation equations, and to normalize the spectrum, it is convenient to introduce the canonical variables $\widehat{\Psi}, \widehat{\Pi}$, whose Fourier modes are defined by

$$
\widehat{\Psi}_{k}=S^{1 / 2} \Psi_{k}, \quad \widehat{\Pi}_{k}=S^{-1 / 2} \Pi_{k},
$$

so that the Hamiltonian density takes the canonical form

$$
H=\frac{1}{2} \sum_{\vec{k}}\left(\left|\widehat{\Pi}_{k}\right|^{2}+k^{2}\left|\widehat{\Psi}_{k}\right|^{2}\right)
$$

Under $S$-duality these canonical variables transform as the original variables. They satisfy Schrödinger-like equations:

$$
\widehat{\Psi}_{k}^{\prime \prime}+\left[k^{2}-\left(S^{1 / 2}\right)^{\prime \prime} S^{-1 / 2}\right] \widehat{\Psi}_{k}=0, \quad \widehat{\Pi}_{k}^{\prime \prime}+\left[k^{2}-\left(S^{-1 / 2}\right)^{\prime \prime} S^{1 / 2}\right] \widehat{\Pi}_{k}=0 .
$$

The amplification of perturbations is typically associated with a transition from an inflationary phase in which the pump field is accelerated (in cosmic time), to a post-inflationary phase in which the pump field is decelerated or constant. In such a class of backgrounds, the "effective potentials" $V_{\Psi}=\left(S^{1 / 2}\right)^{\prime \prime} S^{-1 / 2}, V_{\Pi}=\left(S^{-1 / 2}\right)^{\prime \prime} S^{1 / 2}$, grow (in absolute value) during the phase of accelerated evolution, and decrease in the post-inflationary, decelerated epoch, vanishing asymptotically for very early times $\eta \rightarrow-\infty$ and vanishing again for very late times $\eta \rightarrow+\infty$.

The initial evolution of perturbations, for all modes with $k^{2}>\left|V_{\Psi}\right|,\left|V_{\Pi}\right|$, may be described by the WKB-like approximate solutions of eqs. (3.3)

$$
\begin{aligned}
& \widehat{\Psi}_{k}(\eta)=\left(k^{2}-V_{\Psi}\right)^{-1 / 4} e^{-i \int_{\eta_{0}}^{\eta} d \eta^{\prime}\left(k^{2}-V_{\Psi}\right)^{1 / 2}}, \\
& \widehat{\Pi}_{k}(\eta)=k\left(k^{2}-V_{\Pi}\right)^{-1 / 4} e^{-i \int_{\eta_{0}}^{\eta} d \eta^{\prime}\left(k^{2}-V_{\Pi}\right)^{1 / 2}},
\end{aligned}
$$


which we have normalized to a vacuum fluctuation, and where the extra factor of $k$ in the solution for $\widehat{\Pi}_{k}$ comes from consistency with the first-order equations (2.5). We have ignored a possible relative phase in the solutions. Solutions (3.4) manifestly preserve the $S$-duality symmetry of the equations, since the potentials $V_{\Psi}, V_{\Pi}$ get interchanged under $S \rightarrow S^{-1}$.

In the case of power-law evolution of the pump field, $S^{1 / 2} \sim \eta^{\alpha}$, the effective potentials grow like $\eta^{-2}$ as $\eta \rightarrow 0$, and the approximate solutions (3.4) are valid, at a given time $\eta$, for all modes $k$ with $(k \eta)^{2}>1$. These modes are usually referred to as being "inside the horizon". The corrections to solutions (3.4) are of order $V_{\Psi}^{\prime 2} /\left(k^{2}-V_{\Psi}\right)^{3}, V_{\Psi}{ }^{\prime \prime} /\left(k^{2}-V_{\Psi}\right)^{2}$ and higher, which are indeed small for large $(k \eta)^{2}$. Asymptotically, at $\eta \rightarrow-\infty$, we have $V_{\Psi}, V_{\Pi} \rightarrow 0$ and solutions (3.4) reduce to correctly normalized vacuum fluctuations

$$
\widehat{\Psi}_{k}(\eta)=k^{-1 / 2} e^{-i k \eta+i \varphi_{\vec{k}}}, \quad \widehat{\Pi}_{k}(\eta)=k^{+1 / 2} e^{-i k \eta+i \varphi_{\vec{k}}^{\prime}},
$$

where $\varphi_{\vec{k}}, \varphi_{\vec{k}}^{\prime}$ are random phases, originating from the random initial conditions. Note that because of the random phases, $S$-duality holds only on the average in the sense of eq. (2.7).

For $k^{2}<\left|V_{\Psi}\right|,\left|V_{\Pi}\right|$, equivalently $(k \eta)^{2}<1$ if $S^{1 / 2} \sim \eta^{\alpha}$, the modes are usually referred to as being "outside the horizon". For such modes, it is possible to write "exact" solutions to eq. (3.3) as follows,

$$
\begin{aligned}
& \widehat{\Psi}_{k}(\eta)=S^{1 / 2}\left[\widehat{A}_{k} T \cos \left(S^{-1}, S\right)+\widehat{B}_{k} T \sin \left(S^{-1}, S\right)\right], \\
& \widehat{\Pi}_{k}(\eta)=k S^{-1 / 2}\left[\widehat{B}_{k} T \cos \left(S, S^{-1}\right)-\widehat{A}_{k} T \sin \left(S, S^{-1}\right)\right],
\end{aligned}
$$

where $\widehat{A}_{k}, \widehat{B}_{k}$ are arbitrary integration constants, and the functions $T \cos \left(S^{-1}, S ; k, \eta\right)$, $T \sin \left(S^{-1}, S ; k, \eta\right)$ are defined through the following expansions:

$$
\begin{aligned}
& T \cos \left(S^{-1}, S ; k, \eta\right)=1-k \int_{\eta_{e x}}^{\eta} d \eta_{1} S^{-1}\left(\eta_{1}\right) k \int_{\eta_{e x}}^{\eta_{1}} d \eta_{2} S\left(\eta_{2}\right) \\
& +k \int_{\eta_{e x}}^{\eta} d \eta_{1} S^{-1}\left(\eta_{1}\right) k \int_{\eta_{e x}}^{\eta_{1}} d \eta_{2} S\left(\eta_{2}\right) k \int_{\eta_{e x}}^{\eta_{2}} d \eta_{3} S^{-1}\left(\eta_{3}\right) k \int_{\eta_{e x}}^{\eta_{3}} d \eta_{4} S\left(\eta_{4}\right)+\cdots ; \\
& T \sin \left(S^{-1}, S ; k, \eta\right)=k \int_{\eta_{e x}}^{\eta} d \eta_{1} S^{-1}\left(\eta_{1}\right)-k \int_{\eta_{e x}}^{\eta} d \eta_{1} S^{-1}\left(\eta_{1}\right) k \int_{\eta_{e x}}^{\eta_{1}} d \eta_{2} S\left(\eta_{2}\right) k \int_{\eta_{e x}}^{\eta_{2}} d \eta_{3} S^{-1}\left(\eta_{3}\right) \\
& +\cdots
\end{aligned}
$$


The time $\eta_{e x}$, appearing as an arbitrary lower limit of integration in (3.7), is most conveniently chosen to be near horizon-exit time, i.e. such that $k \eta_{e x} \sim 1$. The above functions satisfy

$$
\left[T \cos \left(S^{-1}, S\right)\right]^{\prime}=-\frac{k}{S} T \sin \left(S, S^{-1}\right), \quad\left[T \sin \left(S^{-1}, S\right)\right]^{\prime}=\frac{k}{S} T \cos \left(S, S^{-1}\right)
$$

Using these relations, and similar ones for $\left[T \cos \left(S, S^{-1}\right)\right]^{\prime}$ and $\left[T \sin \left(S, S^{-1}\right)\right]^{\prime}$, it is possible to verify explicitly that (3.6) are indeed solutions of eqs. (3.3). Formally, these solutions are also valid inside the horizon, but the power series defining $T \cos , T \sin$ are not convergent there. Solutions (3.6) manifestly preserve $S$-duality, which now acts on the parameters of the solutions in the following simple way:

$$
\widehat{A}_{k} \rightarrow-\widehat{B}_{k}, \quad \widehat{B}_{k} \rightarrow \widehat{A}_{k}, \quad S \rightarrow S^{-1} .
$$

We now fix the integration constants by matching solutions (3.4) and (3.6) and their first derivatives at horizon-exit time, for which $k \eta_{e x} \sim 1$. The result (written for the variables without the hats) is simply

$$
\begin{aligned}
& \Psi_{k}(\eta)=\left(k^{2}-V_{\Psi}^{e x}\right)^{-1 / 4}\left[S_{e x}^{-1 / 2} T \cos \left(S^{-1}, S\right) e^{i \varphi_{\vec{k}}}+S_{e x}^{1 / 2} T \sin \left(S^{-1}, S\right) e^{i \varphi_{\vec{k}}^{\prime}}\right], \\
& \Pi_{k}(\eta)=k\left(k^{2}-V_{\Pi}^{e x}\right)^{-1 / 4}\left[S_{e x}^{1 / 2} T \cos \left(S, S^{-1}\right) e^{i \varphi_{\vec{k}}^{\prime}}-S_{e x}^{-1 / 2} T \sin \left(S, S^{-1}\right) e^{i \varphi_{\vec{k}}}\right],
\end{aligned}
$$

where $\varphi_{\vec{k}}$ and $\varphi_{\vec{k}}^{\prime}$ now contain an additional phase depending on the exit time $\eta_{e x}$, and a label $e x$ means evaluation at $\eta=\eta_{e x}$. The above matching respects $S$-duality on the average, in the sense of eq. (2.7).

\section{ENERGY SPECTRA}

To complete a calculation of the energy spectrum of the amplified perturbations we need to perform a second matching step, to late-time solutions after horizon re-entry in the decelerated, post-inflationary era. However, if we are only interested in the leading contribution to the final energy spectrum, we can estimate it by using the well-known phenomenon of 
freezing of perturbations. Solutions (3.10) always contain a time-independent part coming from the first term of the $T$ cos expansion (3.7), and always either the constant part of $\Psi$ or the constant part of $\Pi$ dominates the Hamiltonian in the large wavelength limit $|k \eta| \ll 1$.

In order to illustrate this important point, we parametrize the background during the phase of accelerated evolution with a power-like behaviour of the pumping field, $S^{1 / 2} \sim|\eta|^{\alpha}$ for $\eta<0, \eta \rightarrow 0_{-}$. From the general solution (3.6) we obtain, in the limit $|k \eta| \ll 1$,

$$
\widehat{\Psi}_{k}=\widehat{A}_{k}|\eta|^{\alpha}+\frac{k|\eta|}{1-2 \alpha} \widehat{B}_{k}|\eta|^{-\alpha}, \quad \widehat{\Pi}_{k}=k\left(\widehat{B}_{k}|\eta|^{-\alpha}-\frac{k|\eta|}{1+2 \alpha} \widehat{A}_{k}|\eta|^{\alpha}\right),
$$

with logarithmic corrections for $\alpha= \pm 1 / 2$. Computing the Hamiltonian density (3.2) we obtain, for $|k \eta| \ll 1$,

$$
\begin{aligned}
H & =\frac{1}{2} \sum_{\vec{k}} k^{2}\left\{\left|\widehat{A}_{k}\right|^{2}|\eta|^{2 \alpha}\left[1+O\left(|k \eta|^{2}\right)\right]+\left|\widehat{B}_{k}\right|^{2}|\eta|^{-2 \alpha}\left[1+O\left(|k \eta|^{2}\right)\right]+O\left(\left|\widehat{A}_{k}\right|\left|\widehat{B}_{k}\right||k \eta|\right)\right\} \\
& \simeq \frac{1}{2} \sum_{\vec{k}} k^{2}\left\{\left|\widehat{A}_{k}\right|^{2}|\eta|^{2 \alpha}+\left|\widehat{B}_{k}\right|^{2}|\eta|^{-2 \alpha}\right\} .
\end{aligned}
$$

Thus, irrespectively of the value and sign of $\alpha$, the joint contribution of the constant modes $\Psi_{k} \sim \widehat{A}_{k}$ and $\Pi_{k} \sim k \widehat{B}_{k}$ always provides an accurate estimate of the leading contribution to the Hamiltonian for super-horizon wavelengths. Which of the two constant modes in (4.2) gives the dominant contribution varies from case to case. Also, it is not always true that the subleading constant mode provides the leading correction.

The above statements can be explicitly checked when the pump field $S$ is given by a power of $\eta$ with different powers in the different cosmological epochs. In this case, the joining of solutions at the transition times can be done analytically, exact formulae for the Bogoliubov coefficients can be given, and their duality can be directly checked [12]. An important point [12] is that, for backgrounds with discontinuous derivatives at the transitions, exact duality is satisfied only if continuity is imposed on the original perturbation (and on its first derivative) and not on the rescaled fields $\widehat{\Psi}, \widehat{\Pi}$.

Up to numerical factors, the contributions of $V_{\Psi}^{e x}, V_{\Pi}^{e x}$ in 3.10 can be neglected, and one can thus approximate the normalized vacuum fluctuations outside the horizon by truncating to 1 the $T$ cos expansion and to 0 the $T$ sin expansion, 


$$
\Psi_{k}(\eta)=\left(k S_{e x}\right)^{-1 / 2} e^{i \varphi_{\vec{k}}}, \quad \Pi_{k}(\eta)=\left(k S_{e x}\right)^{1 / 2} e^{i \varphi_{\vec{k}}^{\prime}}
$$

This truncation reflects just a remnant of $S$-duality, the transformation $S_{e x} \rightarrow S_{e x}^{-1}$. It is adequate for an approximate computation of the energy spectrum after re-entry.

At late times $\eta \rightarrow+\infty$, when $V_{\Psi}, V_{\Pi} \rightarrow 0$ in the post-inflationary phase, the perturbation modes with $k \eta \gg 1$ oscillate freely inside the horizon, according to the equations

$$
\widehat{\Psi}_{k}^{\prime \prime}+k^{2} \widehat{\Psi}_{k}=0, \quad \widehat{\Pi}_{k}^{\prime \prime}+k^{2} \widehat{\Pi}_{k}=0,
$$

with plane-wave solutions

$$
\begin{aligned}
& \widehat{\Psi}_{k}=\frac{1}{\sqrt{k}}\left(C_{k} e^{-i k \eta}+D_{k} e^{i k \eta}\right), \\
& \widehat{\Pi}_{k}=\widehat{\Psi}_{k}^{\prime}-\frac{S^{\prime}}{2 S} \widehat{\Psi}_{k} \simeq \widehat{\Psi}_{k}^{\prime}=i \sqrt{k}\left(-C_{k} e^{-i k \eta}+D_{k} e^{i k \eta}\right) .
\end{aligned}
$$

The matching of these solutions to the approximate solutions (4.3), performed at $\eta=\eta_{r e} \sim$ $k^{-1}$, imposes the conditions

$$
\widehat{\Psi}_{k}\left(\eta_{r e}\right)=\frac{1}{\sqrt{k}}\left(\frac{S_{e x}}{S_{r e}}\right)^{-1 / 2} e^{i \varphi_{\vec{k}}}, \quad \widehat{\Psi}_{k}^{\prime}\left(\eta_{r e}\right)=\sqrt{k}\left(\frac{S_{e x}}{S_{r e}}\right)^{1 / 2} e^{i \varphi_{\vec{k}}^{\prime}},
$$

from which we obtain $C_{k}, D_{k}$. The final amplified vacuum fluctuation amplitude, in the regime $k \eta \gg 1$, is thus given by

$$
\begin{aligned}
& \widehat{\Psi}_{k}(\eta)=\frac{1}{\sqrt{k}}\left[\left(\frac{S_{e x}}{S_{r e}}\right)^{-1 / 2} \cos (k \eta) e^{i \varphi_{\vec{k}}}+\left(\frac{S_{e x}}{S_{r e}}\right)^{1 / 2} \sin (k \eta) e^{i \varphi_{\vec{k}}^{\prime}}\right] \\
& \widehat{\Pi}_{k}(\eta)=\sqrt{k}\left[\left(\frac{S_{e x}}{S_{r e}}\right)^{1 / 2} \cos (k \eta) e^{i \varphi_{\vec{k}}^{\prime}}-\left(\frac{S_{e x}}{S_{r e}}\right)^{-1 / 2} \sin (k \eta) e^{i \varphi_{\vec{k}}}\right]
\end{aligned}
$$

(we have absorbed into $\varphi_{k}$ and $\varphi_{k}^{\prime}$ an additional phase factor $k \eta_{r e}$, arising from the matching).

The corresponding averaged Hamiltonian density, for a mode $k$,

$$
\left\langle H_{k}\right\rangle=\frac{1}{2}\left(\left\langle\left|\widehat{\Pi}_{k}\right|^{2}\right\rangle+k^{2}\left\langle\left|\widehat{\Psi}_{k}\right|^{2}\right\rangle\right)=k\left(\frac{S_{e x}}{S_{r e}}+\frac{S_{r e}}{S_{e x}}\right),
$$

is invariant under $S_{e x} \rightarrow S_{e x}^{-1}, S_{r e} \rightarrow S_{r e}^{-1}$, and also under an overall rescaling of $S$. The same invariances thus characterize the spectral energy distribution, $d \rho_{k} / d \ln k=\left(k^{3} / a^{4}\right)\left\langle H_{k}\right\rangle$, which can be written in terms of the proper energy $\omega=k / a$ as 


$$
\frac{d \rho(\omega)}{d \ln \omega}=\omega^{4}\left[\frac{S_{e x}(\omega)}{S_{r e}(\omega)}+\frac{S_{r e}(\omega)}{S_{e x}(\omega)}\right] \simeq \omega^{4} \operatorname{Max}\left\{\frac{S_{e x}}{S_{r e}}, \frac{S_{r e}}{S_{e x}}\right\} .
$$

The approximate expression (4.9) reproduces, up to numerical factors $O(1)$, the leading contribution to all known particle spectra for various models of cosmological evolution. Consider, for instance, graviton production in a transition at $\eta=\eta_{*}$ from de Sitter inflation to radiation, at constant dilaton. In that case $S=a^{2}, S_{e x} / S_{*}=\left(k \eta_{*}\right)^{2}=\left(k / k_{*}\right)^{2}$ and $S_{*} / S_{r e}=\left(k / k_{*}\right)^{2}$, where $k_{*}=\eta_{*}^{-1}$ is the maximal amplified frequency. Therefore $S_{r e} / S_{e x}=$ $\left(k_{*} / k\right)^{4}$. For all modes $\omega<\omega_{*}$, where $\omega_{*}=k_{*} / a, \operatorname{Max}\left\{S_{e x} / S_{r e}, S_{r e} / S_{e x}\right\}=S_{r e} / S_{e x}=$ $\left(\omega_{*} / \omega\right)^{4}$, and we recover the well-known flat Harrison-Zeldovich spectrum [2], $d \rho / d \ln \omega \simeq \omega_{*}^{4}$.

If, instead, we consider the transition from a phase of dilaton-driven inflation to radiation, typical of string cosmology, we can recover from (4.9) the leading contribution to known spectra for photons [9], gravitons [8] and axions [10, 13, 12]. For heterotic photons, in particular, and more generally for gauge bosons coming from extra dimensions, $S(\eta)=e^{-\phi(\eta)}$ coincides with the real part of the usual $S$-field of string theory, and the leading contribution to the energy spectrum (4.9) is the same for two cosmologies that arrive at the same

final $S$ from above (weak coupling) or from below (strong coupling). In the two cases the energy is predominantly stored in the electric and in the magnetic field, respectively. From the approximate expression (4.9) we can also recover the leading contribution to the energy spectrum for more general classes of backgrounds and types of perturbations [13,12].

\section{LOWER BOUNDS ON ENERGY SPECTRA}

Since the approximate expression for the energy spectra, eq. (4.9), contains, as a direct result of the remnant duality symmetry, a factor of the form $x+x^{-1}$, an interesting consequence of the remnant duality symmetry is the existence of a uniform and model-independent lower bound for the energy spectrum:

$$
\frac{d \rho}{d \ln \omega} \gtrsim \omega^{4}
$$


valid for all models of backgrounds and all types of perturbations: it represents the minimal spectrum corresponding to the production of one particle per mode, per polarization and per unit phase-space volume [14]. For some particular model of background, and for a given type of perturbation, eq. (4.9) can provide a less trivial lower bound, corresponding to a "minimal" spectrum flatter than $\omega^{4}$. In such cases the frequency dependence of the lower bound is, in general, model-dependent.

Consider, in particular, the amplification of tensor perturbations in a string cosmology background in which the initial dilaton-driven phase, with $S \sim \eta$, is followed at $\eta=\eta_{s}$ by a high-curvature string phase, with final transition to the radiation-dominated era at $\eta=\eta_{1}$. During the string phase, a total redshift $z_{s}=\left|a_{1} / a_{s}\right|>1$ is accumulated, the curvature is approximately constant, and the dilaton grows by the ratio $\exp \left(\phi_{1}-\phi_{s}\right) / 2=g_{1} / g_{s}>1$. Equation (4.9) gives, in that case:

$$
\frac{d \rho}{d \ln \omega} \simeq \omega^{4} \operatorname{Max}\left\{\frac{\omega_{1}}{\omega}\left(\frac{g_{s}}{g_{1}}\right)^{2} z_{s}^{3}, \frac{\omega}{\omega_{1}}\left(\frac{g_{1}}{g_{s}}\right)^{2} z_{s}^{-3}\right\}, \omega<\omega_{s} \equiv \omega_{1} / z_{s}
$$

The assumption that the pump field keeps growing during the string phase, $z_{s} g_{s} / g_{1}>1$, then leads to the well-known cubic $(n=4)$ graviton spectrum [8], whose lower bound,

$$
\frac{d \rho}{d \ln \omega} \gtrsim \omega_{1}^{4}\left(\frac{g_{s}}{g_{1}}\right)^{2}\left(\frac{\omega}{\omega_{s}}\right)^{3}
$$

is more constraining than eq. (5.1). The "minimal" spectrum is $d \rho / d \ln \omega=\omega_{1} \omega^{3}$ and corresponds to the limiting case in which $g_{s} \rightarrow g_{1}$ and $z_{s} \rightarrow 1$, namely to a model with almost no intermediate string phase. Note that, for gravitons, the largest subleading contribution is not given by the second term in the bracket in eq. (5.2) [8,13] but, instead, can be extracted by carefully matching solutions at $\eta=\eta_{1}$. When this is done, the more stringent bound can be seen as a consequence of a remnant symmetry acting on both $z_{s}$ and $g_{s} / g_{1}$.

An important point is that the duality-symmetric form (4.9) of the spectrum, which provides an accurate order-of-magnitude estimate for the energy spectra produced from perturbations whose action is given by (1.1), can also provide a lower bound on the total energy density of long-wavelength perturbations, even when the background evolution includes a 
high-curvature phase in which the action and perturbation equations are not known. The argument goes as follows.

The result (4.9) was obtained by approximating the evolution of $\Psi$ and $\Pi$ by their constant modes outside the horizon. The approximation was motivated by the explicit solution of the perturbation equations. When the background enters a high-curvature phase, which requires a corrected effective action for an accurate description, the exact form of the perturbation equations and their solutions are not always known, or in any case difficult to obtain in practice. We expect that, for any type of action, the perturbation equations should always admit constant solutions whenever spatial gradients are negligible with respect to time derivatives, in agreement with the general physical principle of freezing of perturbations outside the horizon.

For metric perturbations, a formal argument can be given to justify our conjecture. Since the action is invariant under gauge (i.e. coordinate) transformations, a particular set of allowed perturbations are infinitesimal gauge transforms of the background itself. Of course, these are spurious perturbations without any physical content. It is possible, however, to construct physical, large-wavelength perturbations, which approach the infinitesimal gauge transformations in the infinite-wavelength limit. By continuity, these large-wavelength gravitational waves are approximate solutions of the perturbation equations. The amount by which they deviate from exact solutions away from the limit is controlled by the only dimensionless parameter present, i.e. by $k \eta$. We may thus conclude that, at least for metric perturbations, the constant mode in $\Psi$ is always present for perturbations sufficiently outside the horizon. Canonical Poisson brackets then require that the same be true for П. Although we cannot exclude that other modes in $\Psi$ and $\Pi$ will dominate over the constant modes, assuming that this does not happen will provide a lower bound on the amplified spectra in the presence of a high-curvature phase.

For tensor perturbations, this argument has been confirmed within an explicit string theory model of quadratic curvature corrections [15]. The results of a numerical integration show that $\Psi$ becomes frozen outside the horizon even after including the higher-curvature 
terms in the perturbation equations. The final normalized amplitude is larger, by a numerical factor, than the estimate (4.3), so that the spectrum (4.9) indeed represents a lower bound for the total energy density.

\section{CONCLUSION}

We have shown that the equations of cosmological perturbation theory, to lowest order in the strength of perturbations and in their derivatives, are invariant under a duality transformation acting on the classical moduli of the background and interchanging the perturbation

with its conjugate momentum. As a consequence of this invariance, the large-wavelength part of the Hamiltonian is always dominated by the sum of the contributions of the frozen modes of the perturbation and of its conjugate momentum.

The energy spectrum obtained by truncating the solutions of the perturbation equations to the constant modes is characterized by a residual duality symmetry; it reproduces known results of produced particle spectra and can provide a useful lower bound on particle production, when our knowledge of the detailed dynamical history of the background is approximate or incomplete.

\section{ACKNOWLEDGEMENTS}

We are grateful to A. Buonanno, K.A. Meissner and C. Ungarelli for interesting discussions. R. B. is supported in part by the Israel Science Foundation administered by the Israel Academy of Sciences and Humanities. 


\section{REFERENCES}

[1] E. W. Kolb and M. S. Turner, The Early Universe, Addison-Wesley, Redwood City, CA, 1990; A. Linde, Particle Physics and Inflationary Cosmology, Harwood Academic (1990).

[2] V.F.Mukhanov, A.H.Feldman and R.H.Brandenberger, Phys. Rep. 215 (1992) 203.

[3] A. Giveon, M. Porrati and E. Rabinovici, Phys. Rep. 244 (1994) 77.

[4] G. Veneziano, Phys. Lett. B265 (1991) 287.

[5] A. Sen, Int. J. Mod. Phys. A9 (1994) 3707; J. Polchinski, Rev. Mod. Phys. 68 (1996) 1245; J. H. Schwarz, Nucl. Phys. Proc. Suppl. 55B (1997) 1.

[6] E. J. Copeland, J. E. Lidsey and D. Wands, Nucl. Phys. B506 (1997) 407.

[7] L. H. Ford and L. Parker, Phys. Rev. D16 (1977) 1601; M. Gasperini and M. Giovannini, Phys. Rev. D47 (1993) 1519.

[8] R.Brustein, M.Gasperini, M.Giovannini and G.Veneziano, Phys. Lett. B361 (1995) 45.

[9] M. Gasperini, M. Giovannini and G. Veneziano, Phys. Rev. Lett. 75 (1995) 3796;

D. Lemoine and M. Lemoine, Phys. Rev. D52 (1995) 1955.

[10] E. J. Copeland, R. Easther, D. Wands, Phys. Rev. D56 (1997) 874.

[11] M. Gasperini and G. Veneziano, Mod. Phys. Lett. A8 (1993) 3701; J.D. Barrow, J.P. Mimoso and M.R. de Garcia Maia, Phys. Rev. D47 (1993) 3630.

[12] A. Buonanno, K. A. Meissner, C. Ungarelli and G. Veneziano, JHEP01 (1998) 004.

[13] R. Brustein and M. Hadad, Phys. Rev. D57 (1998) 725.

[14] R. Brustein, M. Gasperini and G. Veneziano, Phys. Rev. D55 (1997) 3882.

[15] M. Gasperini, Phys. Rev. D56 (1997) 4815. 